\title{
»Une chose bien remarquable et importante à la République des Lettres« Gabriel Naudé et l'attribution frauduleuse de l'»Imitation de Jésus-Christ« à Jean Gersen
}

Strada m'a dit a moy qu'il estoit tres difficile d'estre parfait historien, voire mesme impossible parce que pour cela il ne faudroit estre ny d'aucun ordre, ny d'aucun party, ny d'aucun pays ny d'aucune Religion si faire se pouvoit. Les Catholiques Romains favorisent leur party, les moines leur ordre, chacun son pays. Si un homme pouvoit estre Athée sans offencer Dieu il seroit fort propre a descrire l'Histoire de tous les Princes qui depuis tant d'années ont miserablement brouillé toute l'Europe par leur ambition sous pretexte de Religion combien qu'ils n'y croient guerres'.

Le syntagme »république des lettres« n'apparaît que rarement dans les textes de Naudé. Une occurrence nous a frappé, dans une lettre à Jacques Dupuy, datée de Rome le 19 février $1641^{2}$, parce qu'elle fait apparaitre toute l'ambiguité de la position du lettré par rapport aux pouvoirs qu'il sert; dans ce texte, en effet, consacré à l'exposé de la découverte que venait de faire Naudé de la falsification du nom de l'auteur de l'»Imitation du Christ« dans des manuscrits qui lui ont été soumis, l'hétéronomie de la fonction lettrée au $\mathrm{XVII}^{\mathrm{e}}$ siècle paraît de manière éclatante, et pourtant, dans cette relation d'étroite dépendance envers le politique, par la compétence proprement lettrée que ce service même requiert, une forme minimale d'autonomie de l'activité lettrée parvient à se déclarer dans la transmission d'informations destinées aux seuls pairs et dont la diffusion auprès de tout autre public, à commencer par celui des acteurs (des »décideurs« politiques), tout en étant nécessaire, doit être strictement limitée et contrôlée. En effet, les informations sur des matières lettrées, dès lors qu'elles mettent en jeu des conflits de pouvoir et d'intérêts - en l'occurrence entre ordres religieux -, peuvent toujours être préjudiciables à celui qui les écrit, et entre autres choses parce qu'elles sont susceptibles de compromettre le ou les »patrons« du destinateur. Mais alors, bien sûr, ce sont les pairs eux-mêmes, ceux auxquels on transmet l'information, qui sont vraiment dangereux, puisqu'ils peuvent faire, volontairement ou non, un usage indiscret des écrits qui leur sont confiés; et il est besoin de leur préciser les modalités d'usage de l'information et du texte, requérant une sourcilleuse gestion de la confidentialité. Ces instructions et avertissements sont rarement suffisants; rien n'est plus volatile que l'écrit; sans doute est-il plus précis de dire que la confidentialité de l'écrit est intrinsèquement menacée par la dynamique générale de publication dans laquelle les

1 Vienne, Bibliothèque d'État, ms 7071, p. 70.

2 Lettres de Gabriel Naudé à Jacques Dupuy (1632-1652), édition critique Phillip J. WoLfE, Edmonton 1982, p. 116. 
lettrés se trouvent pris, et en particulier par le développement des publications donnant accès à un public toujours plus large à des documents internes au monde lettré (lettres semi-privées, mémoires, etc.), comme si en effet le monde lettré cherchait ainsi à produire des représentations de lui-même, au prix inévitable de la transgression des règles de confidentialité, satisfaisant ainsi un goût nouveau du public pour ces aperçus subreptices (mais le plus souvent savamment mis en scène) sur la vie lettrée. Même les auteurs qui affichent l'aristocratisme le plus prononcé, et théorisent la séparation nécessaire de l'espace privé (auquel appartient le cénacle des amis complices) et de l'espace public, sont emportés dans ce mouvement général de publication; ceux que l'on appelle les »libertins « en donnent eux-mêmes l'exemple par le choix assez fréquent de la langue vernaculaire, la recherche de l'imprimé, le désir aussi de voir éclater au grand jour du public des belles lettres les querelles et les conflits internes au monde lettré, érigeant ainsi le public en juge. D'ailleurs, Naudé rompra lui-même sa propre injonction de confidentialité dans l'affaire que l'on va évoquer, comme nous le verrons, provoquant ainsi une querelle publique par le biais de l'imprimé, doublée d'actions en justice$^{3}$. Cette publicité, à laquelle s'exposent les auteurs à travers des publications imprimées, en latin mais aussi dans les langues vernaculaires, montre combien les lettrés cherchent à toucher un public, dont il savent pourtant qu'il est extérieur pour la plus grande part à la république des lettres telle qu'ils la considèrent stricto sensu, dans le cadre d'une anthropologie sociale farouchement élitaire. Mais c'est aussi, d'abord, par ce biais qu'ils commencent à constituer l'ébauche d'une autonomie sociale du champ littéraire, contre l'idéal, pourtant reconduit, d'une clôture sur soi des activités lettrées.

Mais d'abord, il faut insister d'emblée sur la difficulté, l'étroite limitation et pourtant l'existence effective de la liberté d'initiative des lettrés sous l'Ancien Régime, dans leurs propres activités, dès qu'elles touchent aux intérêts des institutions et des pouvoirs établis. C'est, nous semble-t-il, à la rencontre d'une part de ces difficultés et frustrations permanentes et d'autre part d'une aspiration à l'émancipation sociale du travail intellectuel que le syntagme »république des lettres« trouve sa place et son sens; non donc, point du tout, comme une forme d'organisation et de réglementation de la vie lettrée - une telle république des lettres n'est qu'un fantasme de l'historiographie -, mais comme une instance virtuelle invoquée dans les relations savantes, lorsqu'il s'agit d'affirmer la différence et la spécificité propres de l'activité lettrée au sein même des rapports d'assujettissement qui l'attachent aux pouvoirs civils et religieux et de tenter de faire exister une éthique propre à cette activité chaque fois qu'elle parvient, d'une façon ou d'une autre, à réaliser, fût-ce de manière inchoative ou partielle, cette autonomie. Celle-ci n'est pas une fin en soi; elle se fait au nom d'une valeur, celle de »vérité«, héritée de la philosophie et plus largement des pratiques lettrées, une valeur qui ne saurait reconnaître en tant que telle aucune sujétion sociale, et ceci alors même que les vérités particulières, dans les divers champs relevant de la compétence lettrée, ne peuvent être établies, soutenues et communiquées qu'à travers les jeux complexes de leur instrumentalisation par les acteurs sociaux.

3 Voir infra, coda 1. 
Le passage de la lettre qui nous intéresse commence par ces mots, sur lesquels on pourrait s'arrêter longtemps:

Il faut que je vous avertisse d'une chose bien remarquable et importante à la République des Lettres, mais à la charge que vous vous en servirez s'il en est de besoin sans découvrir l'arcanum academicum à cause que Eugène ${ }^{4}$ [le cardinal Bagno] et moi en recevrions ici des reproches et que l'auteur de la fourberie aurait occasion de s'en ressentir contre nous.

Avertissement assez tortueux et ambigu: Naudé ne s'adresse pas à son interlocuteur uniquement pour l'informer d'une »fourberie« des plus intéressantes pour »la République des Lettres«, mais pour qu'il utilise cette information, cependant sans en révéler le fin mot, de nature à compromettre l'informateur et surtout son patron, le très éminent et politique cardinal de Bagno. C'est que le »fourbe« dont il va être question n'est pas le premier venu: il s'appelle Constantino Gaetani (Naudé l'appelle Cajetan), savant abbé bénédictin, auteur de deux ouvrages visant à démontrer, en se fondant notamment sur des manuscrits anciens en sa possession, que l'»Imitation du Christ « fut écrite par le bénédictin Jean Gersen, au XIII ${ }^{\mathrm{e}}$ siècle. Le piquant et le fond de l'affaire est ce »secret académique« qui va être révélé et que le destinataire devra préserver, tout en sachant s'en prévaloir... On comprend très bien que ce secret n'est pas absolu, qu'il peut être partiellement au moins transmis, mais, précisément, comme un secret à conserver, c'est-à-dire, en clair, qu'il ne doit pas être publié; il peut circuler dans l'espace du cabinet Dupuy, et même en dehors, nous le verrons, jusqu'au cabinet de Richelieu, mais il ne doit pas sortir au grand jour, en particulier sous une forme imprimée. Naudé décrit le contexte de cette affaire, et montre quelle en est l'importance, non seulement pour la république des lettres, mais aussi et même d'abord pour Richelieu, qui venait de créer la Manufacture royale d'imprimerie au Louvre, et ambitionnait d'y développer une prestigieuse politique d'édition, et qui avait précisément choisi pour lancer l'opération l'»Imitation«, best-seller de la littérature dévotionnelle, dont le succès était entretenu par les polémiques au sujet de son auteur véritables.

L'abbé Constantin ou les Bénédictins de Paris à son nom avaient prié M. le Cardinal Duc que puisqu'on imprimait au Louvre le livre De imitatione avec toutes les circonspections possibles pour faire quelque chose de beau et bien entendu, il lui plût ordonner que suivant la vérité découverte par ledit Père Constantin et la foi des anciens MS, il ne fût point publié sous le nom de Thomas à Kempis mais de Joannes Gersen abbas vercellensis etc.

Richelieu ne pouvait ignorer l'existence de trois ou même quatre camps engagés dans une lutte sans merci pour l'attribution de l'ouvrage fameux: celui qui pensait démon-

4 Dans sa correspondance avec les Dupuy, Naudé change systématiquement la plupart des noms de personnages importants et des lieux majeurs selon un code fixe; mesure de protection minimale en vérité, car la restitution des identités ne pose aucun problème sérieux.

5 Selon Richelieu, l'objectif de cette imprimerie est de »Multiplier les belles publications utiles à la gloire du roi, au progrès de la religion et à l'avancement des lettres«. À noter qu'il y publie son propre ouvrage, »L'Instruction du chrétien«, l'année même où paraît l'»Imitation«. 
trer la paternité de Thomas a Kempis, celui qui tenait Jean Gerson pour l'auteur et celui qui affirmait que l'œuvre était plus ancienne et sortie de la plume d'un Jean Gersen, Joannes Gersenius de Canabaco, abbé bénédictin de Vercelli au XIII ${ }^{\mathrm{e}}$ siècle, nom souvent lu dans les manuscrits, prétendument à tort, comme celui du célèbre chancelier de l'université de Paris. Certains, enfin croyaient en la paternité de Bernard de Clairvaux, dont le nom figurait sur certains manuscrits. La querelle, déjà ancienne, mobilisait, on ne s'en étonnera guère, les ordres des religieux qui avaient un intérêt direct dans l'affaire: en particulier les chanoines réguliers de Saint-Augustin, engagés pour leur condisciple Thomas a Kempis, et les bénédictins, tenants de Jean Gersen pour la même raison. Mais, à la différence des précédentes, il faut bien dire tout de suite que cette attribution était nouvelle, qu'elle avait un »inventeur«, en la personne de Gaetani. Gerson avait pour lui les docteurs de Sorbonne et il était le candidat élu de la mouvance gallicane, voire le héros naturel des bons patriotes. Richelieu connaissait sans doute toutes ses prétentions; pour des raisons politiques évidentes, il aurait d'abord penché pour Gerson6; cependant les prières et les raisons des bénédictins ne furent pas sans effet, s'il est vrai qu'il se montra disposé à accepter le nom de Gersen »moyennant toutefois qu'ils fissent venir une foi de Rome comme les MS de ladite ville portaient tous le nom de Gersen et non pas Kempis, à quoi pour obéir les Bénédictins d'ici prièrent Son Eminence de vouloir faire ladite foi«. Richelieu voulait donc une attestation romaine, à laquelle il n'aurait pas manqué de renvoyer les contestataires. Il fit plus, car, si la lettre de Naudé n'est pas claire sur ce point, le résumé de l'affaire qu'il exposa à son ami Guy Patin après le retour en France est sans équivoque:

Il [Richelieu] leur accorda ce qu'ils demandoient a la charge que cela seroit bien prouvé et averé par gens de bien et a ce cognoissans. Il en escrivit a nostre Cardinal Bagno qui, comme il estoit homme d'esprit, se fit apporter les 4 Manuscrits dont les Benedictins estoient ravis pensant le tromper, mais ne le purent, car il nous les fit examiner tous devant luy, et fort particulierement, et y trouvames 7 tout falsifié et raturé8.

6 C'est ce qu'il est possible de déduire de la lettre suivante de Naudé à Dupuy, du 28 avril, WOLFE (éd.), Lettres de Gabriel Naudé (voir n. 2), p. 119.

7 Intéressante la correction dans le manuscrit de »trouvames« en »trouva«, exigée par la grammaire, mais signifiante.

8 Vienne, Bibliothèque d'État, ms 7071, p. 60. La saisie des notes est probablement de Patin, mais il a indiqué le jour où il s'est entretenu avec Naudé: le 12 mars 1642, date qui fait problème car certaines informations sont un peu ultérieures dans le temps. Ce passage, comme bien d'autres, par sa proximité avec ce que Naudé a écrit par ailleurs et sa forme d'énonciation, atteste que toutes ou partie de ces informations viennent directement de l'ami de Patin. Nous donnons ici l'ensemble de la notice, indiquée en marge »quis autor libri de imitatione Christi«: $»$ Le livre de Imitatione christi a pour vray Auteur Thomas a Kempis chanoine Regulier de Flandres, et cela est tres certain. Les Benedictins voudroient bien que le monde crûst que l'Auteur fust un certain des leurs qu'ils nomment Joannes a Gersen qui a esté un Abbé Benedictin. De là vient qu'on dit en France que c'est Jean Gerson (qui fut il y a plus de 200 ans un celebre Docteur de Sorbonne et chancelier de l'Université de Paris!) per regulam de duobus litigantibus gaudet tertius. On le trouve de vieille edition sous ce nom de Gerson. Je l'ay vû aussi sous le nom de S. Bernard. Mr l'Abbé l'Advocat travaille sur cette matiere et veut prouver pour l'honneur de la France que le vray Auteur de ce livre est ce Jean Gerson. Mais il n'en 
Richelieu ne voulait pas une attestation romaine quelconque, mais l'avis d'une autorité ecclésiastique en laquelle il avait entièrement confiance, et qui était aussi un lettré averti, entourée de doctes experts en livres et manuscrits. Les bénédictins, aux dires de Naudé, avaient mal évalué les exigences de l'interlocuteur imposé par Richelieu (peutêtre fut-il d'ailleurs mis en alerte par Richelieu lui-même): le procureur à Rome de la congrégation de Saint-Maur, Dom Placide Simon, et le procureur des bénédictins anglais, Dom Jean Rubeus, se déplacèrent en personne et

apportèrent 4 exemplaires du livre De imitatione, dont les 3 étaient MS et le $4^{\mathrm{e}}$ imprimé sous le nom de Joannes Gerson chancelier de Paris. Mais d'autant qu'à la fin d'icelui il y avait une note écrite à la main qui disait snota quod liber huius autor non est Joannes Gerson sed Joannes ... abbas vercellensis ut apparet< etc., ils prétendirent que s'en servir comme d'un MS et creurent que Son Emine Eugène après les avoir vus alla buona commanderait à son secrétaire de faire l'attestation qu'ils désiraient'.

Il est vrai que donner un ouvrage imprimé pour un manuscrit à cause de la présence d'une note manuscrite augurait mal du sérieux des religieux en question. Quoi qu'il en soit, Bagno, loin de s'empresser de céder à la requête des bons pères, exigea une véritable expertise, en bonne et due forme, réalisée par deux spécialistes: Fioravante Martinelli, ex-bibliothécaire de la Vaticane, connaisseur des »antichità dei tempi mezzani« ${ }^{10}$, et Naudé lui-même:

L'affaire alla autrement, car Eugène voulut que ces livres fussent visités par deux personnes capables d'en pouvoir juger et qu'ils en fissent leur rapport en sa présence, ce qui leur donna occasion de me nommer pour l'un d'iceux et un nommé Fioravantes Martinellus pour l'autre. Ensuite de quoi ils allèrent par ordre de Eugène chez son notaire faire dresser l'instrument à notre relation près.

viendra jamais a bout. Le Cardinal de Richelieu faisant rimprimer ce livre au louvre avoit resolu d'y faire mettre le nom de Thomas a Kempis. [mg: Thomas a Kempis vivoit il y a environ 200 ans]. Les Benedictins de France intervinrent et le prierent d'y mettre le nom de Jo. Gersein se vantans d'avoir pour le prouver quatre manuscrits de ce livre a Rome qui tous 4 portoient ce nom. Il leur accorda ce qu'ils demandoient a la charge que cela seroit bien prouvé et averé par gens de bien et a ce cognoissans. Il en escrivit a nostre Cardinal Bagno qui, comme il estoit homme d'esprit, se fit apporter les 4 Manuscrits dont les Benedictins estoient ravis pensant le tromper, mais ne pûrent, car il nous les fit examiner tous devant luy, et fort particulierement, et y trouvames tout falsifié et raturé. Ce qui estant mandé par luy de deça on n'a mis, a cause de l'incertitude en laquelle ils sont demeurez le nom d'aucun Auteur a l'edition du louvre. Nous verrons ce qu'en dira quelque jour Mr l'Abbé l'Advocat au livre qu'il en fait en faveur de Jean Gerson."

9 Wolfe (éd.), Lettres de Gabriel Naudé (voir n. 2), p. 116. Dans sa lettre du 28 avril, Naudé dit encore que Gaetani »ne conçut pas que notre cardinal [Bagno] y dût apporter tant de circonstances, car en effet ces bons pères qui les apportèrent ne désirèrent autre chose sinon deux mots d'attestation comme l'écriture en était fort ancienne«, Ibid., p. 119.

10 Selon ce qu'affirme Naudé lui-même dans sa lettre à Dupuy du 28 janvier 1639, Wolfe (éd.), Lettres de Gabriel Naudé (voir n. 2), p. 70. 
On notera évidemment le souci, qui aura des conséquences considérables dans la querelle, de faire appel à un notaire pour dresser un acte attestant la présence du nom de Gersen. Les bénédictins d'ailleurs, une fois les falsification mises en évidence, renverront le notaire, pour éviter de souscrire ainsi à leur propre défaite. Le notaire avait cependant établi une première description des livres, avant leur examen, et ce document va conférer au rapport de Naudé, établi le même jour, un statut semi-juridique dont ce dernier se prévaudra, et au moins pourra-t-il se défendre ainsi de l'accusation d'avoir falsifié lui-même les documents (voir infra).

La description du processus d'expertise est très circonstanciée et, il faut en convenir, fort probante: sous des ratures, Naudé vit apparaître le nom de »Thomas" et aperçut une substitution de titres; une miniature représentant un religieux bénédictin indiquait le monastère de provenance de la copie et non l'auteur; un manuscrit réputé plus ancien que l'époque où vécut Thomas a Kempis ne l'était nullement, puisqu'on y trouvait aussi, de la même plume, le texte d'une bulle de $14488^{11} \ldots$ Bref, aucun des indices censés démontrer la paternité de Jean Gersen n'était probant. Bien au contraire, les documents portaient des signes manifestes de falsification. »Ce qu'ayant montré et fait voir et juger à l'œil et au doigt à Eugène, il demeura bien étonné de la tromperie«. Le cardinal Bagno constata et attesta de son autorité la justesse de l'expertise naudéenne devant les religieux qui

s'en retournèrent confus, repentis, et abjects, disant que l'abbé Constantin, à qui trois de ces livres appartenaient, ferait venir l'attestation d'autres MS qui étaient en Allemagne pour légitimer ce pseudo Gersen. À quoi je vous supplie de vous opposer pour la vérité, s'il en est de besoin, mais néanmoins sans faire mention de cette histoire si ce n'était à Son Eminence Ducale et ce encore en la suppliant de se servir de l'avis sans vouloir découvrir d'où il vient, car autrement vous me mettriez en un grand labyrinthe et Eugène peut-être aussi en recevrait aussi quelque déplaisirir ${ }^{12}$.

Les bénédictins n'avaient donc pas dit leur dernier mot, et Naudé réitère sa demande d'aide, au nom de la »vérité«, pour dissuader Richelieu d'accéder à la requête des religieux, mais aussi, comprend-on, pour faire courir le bruit, sans dévoiler l'arcanum academicum, que Gaetani est un faussaire et l'attribution de l'»Imitation« à Gersen une fiction.

Sa lettre suivante, au même destinataire, du 28 avril, est également très riche d'enseignements. D'abord, elle montre d'entrée de jeu que les procédures de réserve et de secret ne sont pas sans effets: Dupuy, pourtant mieux informé que quiconque, ignorait tout de la demande d'attestation romaine, de même que Naudé dit ne pas avoir pris jusque-là la mesure du conflit parisien entre bénédictins et réguliers de Saint-Augustin. Il propose alors à son interlocuteur de faire tout simplement triompher les seconds en lui envoyant, jointes à sa lettre, des »écritures«, car au moins apparaitra-t-il que le bon abbé Joannes Gersen a été supposé par les bénédictins. Parmi ces écritures figure sans

11 WOLFE (éd.), Lettres de Gabriel Naudé (voir n. 2), p. 117. Voir le rapport publié en 1647 cité en annexe, plus précis encore que ne l'est la lettre à Dupuy.

12 Ibid. 
nul doute le rapport de Naudé, faisant suite à la description des manuscrits établie par le notaire de Bagno, qui sera publiée par Jean Fronteau quelques années plus tard ${ }^{13}$. Surtout, il fait valoir des arguments contre la publication de l'»Imitation« sous le nom de Gerson, qui semble tenter Richelieu, mais aussi son interlocuteur, car »ce serait établir une fausseté déjà reconnue et avouée de tout le monde, cela témoignerait un amour trop flatteur de notre patrie $\ll^{14}$. En cela, la manœuvre serait non pas utile, mais dommageable pour l'Imprimerie royale, car tous les ouvrages qu'elle ferait paraître, après ce malheureux coup d'essai, s'en trouveraient discrédités. Du reste, le calcul est simple: si l'on met le nom de Gerson, il faudra se défendre contre »trois partis différents et déjà tout formés«, alors que si l'on opte pour Thomas a Kempis, qui, selon Naudé, est le véritable auteur, on peut s'appuyer sur des ouvrages composés par des "personnes indifférentes « - les jésuites Henri de Sommal et Herbert Rosweyde ${ }^{15}$ - et seuls les bénédictins protesteront, mais il joint à sa lettre de quoi leur clouer définitivement le bec. Naudé envisage donc bien une publication de son rapport, mais dont les »messieurs« de l'Imprimerie, sinon Richelieu lui-même prendraient l'initiative, sans doute en le joignant à l'édition, comme Rosweyde l'avait fait en accompagnant le texte d'une réfutation en règle de Gaetani. Le ton de Naudé à l'égard du très respecté Dupuy se fait même très directif: »Monsieur, autant que vous avez le zèle pour l'honneur de la France et celui de ces Messieurs qui ont fait dresser cette imprimerie, comme encore pour la vérité, autant me semble-t-il que vous devez faire votre possible pour faire entendre ces vérités à celui qui en a la surintendance et lui remontrer les inconvénients qui pourront arriver de les avoir négligées«. En matière de livres, le politique a en effet tout intérêt à se plier aux conseils du lettré, sans quoi il risque fort de susciter des conflits interminables, de se discréditer et pis encore de se ridiculiser. Cette assurance est évidemment digne d'être soulignée: tout ministre d'État qu'il soit, Richelieu ne peut impunément faire mettre en tête du plus connu des ouvrages de spiritualité un nom choisi pour sa seule efficacité idéologique. Autrement dit, il est dans son intérêt politique même de faire valoir, en la matière, »la vérité«, telle qu'elle est établie par des experts non partisans (des jésuites et Naudé), et cela veut dire qu'il doit se soumettre au verdict d'une »vérité« extérieure à la sphère politique, établie par des lettrés dotés d'une autorité scientifique. De ce point de vue, la république des lettres entendue comme la communauté virtuelle de tous ceux qui ne reconnaîtraient comme »vérité« que celle qui est établie par leurs pairs, selon des critères partagés de rationalité, pos-

13 Voir infra, coda 1.

14 WolfE (éd.), Lettres de Gabriel Naudé (voir n. 2), p. 118.

15 De imitatione Christi libri quatuor. Authore Thoma a Kempis, canonico regulari, ordinis D. Augustini; ad autographum emendati, opera ac studio Henrici Sommalii [Henri de Sommal] è Societate Jesu, Berg, Veuve d'Adam, 1612; Thomae a Kempis canonici regularis ord. S. Augustini De Imitatione Christi libri quatuor; nunc postremò ad autographorum fidem recensiti. Cum Vindiciis Kempensibus Heriberti Ros-weydi [Herbert Rosweyde] Soc. Jesu, adversus C. Caietanum abbatem S. Baronti. Ad. S. D. N. Paulum V, Antverpiae, ex officina Plantiniana, apud Balthasarem \& Joannem Moretos. 1617. Comme l'indique le titre, celui-ci réagit, dans le pamphlet qui accompagne l'édition (Vindiciae Kempenses), à l'attribution de Caietano, publiée en 1616. 
sède bien aux yeux de Naudé un pouvoir effectif, en son domaine propre, sur l'acteur politique lui-même. Mais on peut remarquer aussi que le lettré n'attend pas qu'un consensus soit établi dans la république des lettres pour rechercher une validation et une caution des autorités, ou au moins une permission et une tolérance, et cela indique bien qu'il en attend une action efficace en vue même de l'obtention d'un tel consensus. Car Naudé sollicite de la part de l'autorité (pour le coup, à la fois ecclésiastique et politique) l'arbitrage du conflit existant, par la reconnaissance de la »vérité« dont il apporte les preuves, qui sont celles de sa science des livres, mais en tant qu'elles ont été constatées par une autorité morale (Bagno) et une caution juridique (présence du notaire et des témoins). Le processus - de »vérification« et de »véridicité« - qui conduit à établir et à faire reconnaître une vérité comme telle est en fait un montage où le lettré est, dans ses actes d'expertise et d'écriture, de bout en bout en situation d'hétéronomie. Certes, en faisant publier le texte de l'»Imitation« sans nom d'auteur, Richelieu, à travers la médiation de Dupuy (il faudrait s'arrêter sur ce rôle16), semble s'être en partie incliné devant la »vérité « du lettré, confortée par l'autorité d'un membre éminent de la curie romaine, mais en partie seulement, car il a d'abord tenu compte, par son choix, du dissensus persistant des doctes ${ }^{17}$. Notons d'ailleurs que l'histoire donne raison à la prudence toute politique du cardinal, s'il est vrai que la querelle de l'»Imitation « se poursuit encore aujourd'hui ${ }^{18}$.

Devant cette demi-victoire du lettré, on voit très bien comment ce qu'il tient pour une »vérité« méritant d'être reconnue par la république des lettres ne peut s'imposer que dans la mesure où elle est validée par les puissances et les institutions de la »république« réelle et non du seul fait du consensus des doctes (d'ailleurs plus souvent affirmé que réellement atteint). L'activisme de Richelieu pour contrôler la production

16 Il nous semble utile à ce propos de discuter ce qu'écrit Orest RANUM, au sujet de Jacques Dupuy et de son rapport à la république des lettres, mais aussi, par la même occasion, au sujet de Naudé: »Une si forte identité de juriste au service de l'État laissait peu de place à la réflexion ou au rêve d'être un citoyen de la république des lettres. Comme Naudé l'a écrit à Jacques Dupuy: >je suis sujet de Sa Majesté et serviteur du Cardinal de Bagny, auquel et le Roi et M. le Cardinal de Richelieu portent tant d'affection<. Tout le reste est littérature«, dans: »La Bibliothèque du Roi, Foyer de la République des Lettres ?«, http://www.ranumspanat.com $/ \mathrm{html}$ pages/republic_letters.html (30/03/10). Or dans cette lettre du 10 octobre 1637 (WoLfE [éd.], Lettres de Gabriel Naudé, p. 39), Naudé, demande à Dupuy d'intercéder auprès de Richelieu et de Séguier pour un éventuel emploi, mais à la fois l'entretient, comme à l'accoutumée, de nouvelles de livres et en particulier de son »Syntagma de studio militari«, dont il demande précisément à Dupuy qu'il en donne des exemplaires aux deux politiques. Ce faisant, il est dans une posture de service, certainement, jusque dans la composition de ce type d'ouvrage (qui visent à servir aux praticiens du politique), mais l'investissement dans le travail d'érudition ne saurait d'aucune façon se résumer ou se réduire à ce service politique. Aussi ne peut-on dire que »tout le reste est littérature«, parce que la »littérature« est présente dans la relation politique ellemême, sans s'y limiter pour autant.

17 De imitatione Christi, libri IV, Parisiis anno MDCXL e Typographia regia. On notera cependant que le texte édité est celui qui a été établi par Herbert Roweyde, kempiste résolu. L'ouvrage est daté de 1640, mais semble être sorti l'année suivante. Il serait important d'établir une date précise pour mesurer l'efficacité de Naudé.

18 Voir coda 2. 
culturelle en créant des lieux institutionnels de la culture immédiatement inféodés au pouvoir d'État est bien connu (création de l'Académie française, etc.). Il a bien sûr à voir avec la conscience politique de la montée en puissance du (contre-)pouvoir potentiel des lettrés, qu'il s'agit de neutraliser et d'amener à servir les intérêts de l'État en général et la politique du cardinal en particulier. Mais la création de la manufacture royale d'imprimerie, les difficultés qu'elle rencontre immédiatement et, il faut bien le dire, sa relative stérilité, montrent bien aussi que cette captation politique de la république des lettres ne va pas de soi. La politique éditoriale de l'Imprimerie est axée sur le prestige, dans la présentation d'œuvres déjà consacrées, dont le profit idéologique semble évident, et cet esprit est aux antipodes de la conception qu'un Naudé, par exemple, se fait des priorités éditoriales: donner accès à des textes introuvables, insuffler des recherches nouvelles... Aussi l'érudit se montre-t-il extrêmement critique à l'égard de l'Imprimerie dans cette lettre à Dupuy et en d'autres ${ }^{19}$. Cela atteste aussi, nous semble-t-il, que le pouvoir d'État d'une part, dans ses politiques culturelles, et les lettrés d'autre part, lorsqu'ils travaillent à faire accepter leurs »vérités« par celui-ci en arguant de leur utilité, n'ont pas, et ne sauraient avoir, les mêmes objectifs. En guise d'hypothèse provisoire, nous avancerons que ce que les lettrés appellent république des lettres est cet espace social virtuel structuré selon les propres objectifs, valeurs et priorités des pratiques lettrées saisies dans leur autonomie, en tant qu'ils différent ou divergent des objectifs, priorités et valeurs que poursuivent et reconnaissent ceux qui ont en charge la »république« réelle, et les lettrés eux-mêmes quand ils ont à exercer les fonctions ancillaires du service de plume ${ }^{20}$.

La compétence lettrée dont Naudé peut se prévaloir pour persuader les acteurs politiques de son utilité est, comme on l'a déjà vu, essentiellement historique et bibliographique: il la met ici à l'œuvre pour achever de convaincre et d'instruire son destinataire en reprenant l'affaire de la querelle, depuis les attributions infondées à Gerson jusqu'à l'invention de Gersen par Gaetani. Celui-ci, il faut y insister, n'est pas extérieur à la république des lettres: auteur de divers ouvrages d'érudition religieuse, il en est au contraire un citoyen éminent. Naudé l'avait signalé comme docte dans l'un de ses livres et lui conserve malgré tout une certaine estime au moment même de la que-

19 Voyant que l'on parle d'imprimer les conciles en 40 volumes à l'Imprimerie royale, dans la même lettre, Naudé critique vertement la politique éditoriale de Richelieu: »Combien n'auraitil pas été plus à propos d'employer cet argent à faire un corps grec et latin des interprètes d'Aristote, à augmenter, comme l'on pourrait faire, la Bibliotheca patrium de dix ou douze volumes, à poursuivre les Historiens de la France laissés par feu M. Duchesne et à faire quelque autre chose de semblable, ou au moins à imprimer tant de livres anciens qui ne se trouvent plus, voire même à publier une infinité de bons MS que l'on pouvait tirer de la Bibliothèque du Roi et qui eussent apporté un honneur immortel à notre nation«, WOLFE (éd.), Lettres de Gabriel Naudé (voir n. 2), p. 120. Cf. la lettre du 31 juillet: »la dépense de cette Imprimerie Royale aurait été bien mieux employée à dresser une bibliothèque publique«, ibid., p. 129 .

20 Voir, sur cette notion, les travaux de Christian JouHAUD, en particulier: Sur le statut d'homme de lettres au XVII ${ }^{\mathrm{e}}$ siècle: la correspondance de Jean Chapelain (1595-1674), dans: Annales. Histoire, Sciences sociales 2 (1994), p. 311-347 et ID., Les pouvoirs de la littérature. Histoire d'un paradoxe, Paris 2000. 
relle $^{21}$. Mais ce qui le rend indigne de cette belle république, c'est qu'»il n'a rien plus à cœur que d'étendre per fas comme je crois et nefas l’honneur des Bénédictins «22, disposé qu'il est à attribuer l'»Imitation « à l'un des siens par imposture et falsification. Du reste Gaetani était déjà suspect à Naudé, pour s'être mis en tête de prouver que saint Pierre avait séjourné »dans la même maison où est maintenant le Collège des Bénédictins duquel il est recteur in Trastevere «23. Autrement dit, Gaetani est le type même du lettré falsificateur et affabulateur, et s'il en est ainsi, c'est qu'il met le service de son ordre au-dessus de l'exigence de vérité autour de laquelle la très virtuelle république des lettres se constitue. Et l'on voit bien que le combat met aux prises des lettrés - en tant que tels membres légitimes de la république des lettres - en même temps qu'il oppose les ordres religieux, mais Naudé s'appuie sur la valeur suprême de la république des lettres: la vérité, telle qu'il est possible de la décider par l'enquête historique et philologique rigoureuse au sein d'une embryonnaire communauté de chercheurs reconnaissant les mêmes critères d'évaluation et d'établissement. Et l'on voit aussi par là que la notion de république des lettres est pour lui essentiellement profane, affranchie des intérêts confessionnels et ici monastiques, comme elle l'est, idéalement, des intérêts et conflits politiques. Aussi, est-ce en ce sens qu'il conclut sa mise au point historiographique: „Voici une longue histoire de peu de choses, sed nugae seria ducunt, et j'estime que ce n'est pas peu de choses parmi tant d'impostures qui s'introduisent tous les jours au préjudice de la vérité d'en avoir assurément découvert une comme celle de Joannes Gersen, qui prendra fin dès maintenant si ces Messieurs de l'Imprimerie Royale se veulent servir à leur profit de l'histoire dont je vous ai écrit si amplement par celle-ci et par ma précédente.« Naudé ne cache pas sa jubilation d'avoir confondu une falsification historique au moins parmi celles qui »s'introduisent tous les jours«. »Nugae«, vétilles certes, vétilles d'érudits en l'occurrence, »sed nugae seria ducunt«: mais les vétilles conduisent à des choses graves... Sans qu'il précise plus sa pensée, il semble assez évident que Naudé revient ainsi, in fine, au politique: les querelles et les mensonges des moines peuvent avoir les plus terribles conséquences politiques, comme on l'a vu avec le schisme protestant et les guerres civiles de

21 Panegyricus dictus Urbano VIII Pont. Max., ob beneficia ab ipso in M. Thom. Campanellam collata, Paris, Cramoisy, 1644, p. 90. Mais l'ouvrage avait été composé bien avant l'affaire dont il est ici question. Caietano figure aussi dans les »Apes Urbanae« de Leo Allaci pour ces nombreuses publications et notamment son édition du $»$ De imitatione Christi« parue en 1616, »rendue « à Gersen (multis in locis ex Veteribus Codicibus restitutos, auctos, \& emendatos, quibus addidit, Concertationem, qua verus Auctor, eorundem librorum Ioannes Gersen Abbas Vercellensis vindicatu, Rome, 1633, p. 73). Caietano appartenait aux réseaux lettrés, et entretenait, entre autres, une correspondance avec Peiresc. Naudé, en 1642, juste avant de faire état de la querelle sur l'»Imitation«, le présente à Patin comme »un homme qui scait beaucoup mais avec trop peu de jugement, qui en recompense a beaucoup de feu et un grand esprit«, Vienne, Bibliothèque d'État, ms 7071, p. 59.

22 Wolfe (éd.), Lettres de Gabriel Naudé (voir n. 2), p. 119.

23 Ibid., Lettre à Dupuy, 27 juillet 1638, p. 61 et d'ajouter »Vous direz peut-être que c'est une chimère semblable à l’Épiphanie de M. de Lapeyre. Mais ledit abbé se promet bien le contraire, et dit avoir des témoignages irréfragables pour leur antiquité«, ibid. 
Religion. Du moins, est-ce ainsi que nous nous risquons à développer ici sa pensée. C'est pourquoi, du reste, il trouve tout à fait opportun et même nécessaire une intervention directe des pouvoirs politique et judiciaire pour régler les conflits de plume; lorsque l'affaire rebondira, lui-même y aura recours, obtenant in fine, par sentence du parlement, l'interdiction de publier dorénavant l'»Imitation« sous le nom de Gersen, au profit du seul Kempis ${ }^{24}$. Ce jugement, à lui seul, en dit très long sur les limites de l'autonomie reconnue à la république des lettres, et qu'elle se reconnaît elle-même à travers ses membres. Au cours d'une controverse particulièrement âpre et virulente, Naudé ira jusqu'à invoquer contre Gaetani, désormais décédé, le précédent d'Alfonso Ceccarelli, célèbre faussaire et contrefacteur de livres, supplicié à Rome par Grégoire XIII en 1583. Naudé revient plusieurs fois, et notamment dans sa correspondance avec Dupuy, sur le cas fascinant de Ceccarelli25, mais certainement pas pour mettre en cause la justice ou la légitimité de son exécution, tant il lui paraît évident que les »criminels« de la république des lettres (qui après tout ne font rien d'autre que de brouiller du papier) méritent d'être remis au bras séculier, à la fois pour leurs méfaits dans les lettres et le danger politique qu'ils représentent.

Il serait cependant tout à fait abusif d'en déduire que Naudé milite pour une société civile libérée, par les bons soins d'une justice implacable, des impostures des moines et des faux doctes, car il reconnaît plus qu'aucun autre la nécessité politique de l'imposture: le syntagme »république des lettres« sert ainsi à désigner un espace protégé où la vérité pourrait idéalement circuler en vase clos, sans porter préjudice à la société. Il revient aux acteurs politiques, assistés de leurs conseillers lettrés, d'organiser la répression des impostures délétères et la promotion des impostures utiles, bénéfiques, parfois salvatrices ${ }^{26}$. Dans ce cas, il conseille à Richelieu, par l'intermédiaire de Dupuy, de démasquer publiquement les impostures des bénédictins, car, au contraire, un mensonge, même le pieux mensonge d'une attribution à Gerson, lui serait préjudiciable. Mais il admet par ailleurs que la vérité doive être tue, et même pourchassée lorsqu'elle peut porter préjudice à ceux pour lesquels il travaille, ou, plus généralement, à l'ordre politique ou à l'unité de l'Église. Il est d'ailleurs tout à fait révélateur que le point

24 Voir infra, coda 1.

25 »On réimprime in- $12^{\circ}$ la censure du sieur Leo Allatius sur les Antiquités de Volterre, avec le narré de toutes les faussetés et impostures en semblable matière d'Alfonse Ceccarelli, qui fut pendu ici et eu le poing coupé pour avoir supposé beaucoup de livres, d'histoires, et de privilèges de famille«, 31 juillet 1641, WolfE (éd.), Lettres de Gabriel Naudé (voir n. 2), p. 130. »Ledit sieur [Leone Allacci] nous a depuis déchiffré gentiment toute l'histoire de cet Alfonso Ceccarelli, médecin de Bavagna, qui fut pendu ici il y a quelque cinquantaine d'années pour avoir contrefait une infinité de livres et instruments [...]«, 27 novembre 1641, ibid., p. 133. Cf. ms Patin de Vienne, Bibliothèque d'État, ms. 7071, p. 46. Voir Leone AllacCI, Animadversiones in Etruscarum Antiquitatum fragmenta ab Inghiramio edita, cum Animadversione in Alphonsi Ciccarelli libros, et Auctores ab eo confictos, Paris 1640. Gabriel NAUDÉ y revient dans le Mascurat, Jugement de tout ce qui a esté imprimé contre le Cardinal Mazarin. Depuis le sixieme janvier, jusques à la declaration du premier avril 1649, s.l.n.d., p. 48.

26 On pense ici en particulier aux analyses de Naudé développées dans ses »Considérations politiques sur les coups d'État«, Rome 1639. 
abordé immédiatement après l'affaire de l'»Imitation« soit, dans sa lettre du 10 février, la demande expresse d'intervention commandée par Bagno pour »empêcher la vente« d'un pamphlet contre lui, »Cancellaria bavarica«, qui dénonçait l'alliance que le nonce avait négociée entre la France et la Bavière à l'insu de Rome dix ans auparavant. La meilleure solution, explique Naudé, " pour le respect du maître [Bagno], du père [le roi, ou le pape], et de Viracleo [Richelieu] serait de supprimer tout doucement ces mémoires, ajoutant après qu'il le fallait faire avec grande dextérité, crainte que si l'on savait qu'ils eussent été supprimés en Hollande, on ne les réimprimât en Franconie ou en quelque autre endroit « ${ }^{27}$. Si nous nous contentions de souligner le rôle assumé par Naudé de défenseur et de diffuseur de »vérités « indignement falsifiées, nous n'aurions qu'une vue tout à fait partielle de la réalité: l'une de ses tâches, et il en va de même pour son destinataire, consiste à pourchasser, en mission commandée, des ouvrages au contenu politique compromettant, indépendamment des vérités éventuelles qu'ils contiennent.

Cette attitude complexe, qui trouve ses raisons d'être dans la situation objective du lettré (en l'occurrence un auteur astreint à servir à la fois les intérêts de la France toute monarchique et ceux de la curie romaine), apparaît très bien dans une lettre suivante du 17 mai, au même Dupuy, où Naudé évoque avec admiration le livre »bien hardi et encore mieux raisonné« du père Sirmond, visant à démontrer que saint Denis de Paris et Denis l'Aréopagite étaient distincts ${ }^{28}$, contre la légende de leur identité, alimentée par patriotisme. Naudé se réjouit et souhaite la multiplication de ces initiatives audacieuses: »à l'exemple du Père Sirmond j'espère que beaucoup d'autres galants hommes feront bonne guerre à tant d'autres fables et opinions semblables«. Ces galants hommes sont les héros de la république des lettres qu'il appelle de ses vœux. Lorsqu'il salue l'initiative historiographique de Sirmond et d'autres entreprises du même genre (Jean de Launoy contre le mythe de la présence de Lazare et de Madeleine en Provence, contre Denis de Paris, etc. ${ }^{29}$ ) comme dans son propre combat contre l'attribution patriotique de l'»Imitation« à Gerson, on voit très bien que, comme mem-

27 WolfE (éd.), Lettres de Gabriel Naudé (voir n. 2), p. 118.

28 Dissertatio, in qua Dionysii Parisiensis \& Dionysii Areopagitae discrimen ostenditur, Paris 1641. Jacques Sirmond était jésuite, et l'on pourrait s'étonner de cette fréquente alliance de Naudé avec la compagnie, déjà à l'œuvre dans son ouvrage contre les rose-croix et qui trouve son point d'orgue dans le travail d'éditeur et de préfacier au bénéfice d'ouvrages issus des rangs de la compagnie, visant à appuyer la thèse kempiste dans la querelle de l'»Imitation«. Cependant Naudé se réjouit tout autant des recherches à même de porter atteinte au fondateur et par là au prestige de la Compagnie de Jésus, dont il dit le plus grand mal dans ses conversations avec Patin. Notamment, et cela ne manque pas de sel, il est fort aise de l'ouvrage que Caietano a écrit contre Loyola (Sant Inigo, Venise 1641): »Il prouve en ce livre que Saint Ignace a esté Benedictin premierement [...], qu'il a desrobé son livre d'Exercices Spirituels d'un ancien Benedictin, que jamais ce pere Ignace n'a fait de miracles, qu'il ne devoit pas estre canonisé, qu'il estoit meschant homme, qu'il avoit esté repris pour sodomie«, Vienne, Bibliothèque d'État, ms 7071, p. 59.

${ }_{29}$ Cf. lettre à Doni du 18 avril 1642, fonds It. Ms 1671, fol. 13r. Sur l'intense activité de »chasseur« de saints de Launoy, voir A. PINTARD, Le libertinage érudit dans la première moitié du XVII ${ }^{\mathrm{e}}$ siècle, Paris, 1943, p. 279-280. Cela ne l'empêchera pas de s'opposer à Naudé dans la querelle sur l'»Imitation«, prolongeant la querelle au-delà de l'arrêté du parlement. 
bre de la république des lettres épris de vérités historiques, Naudé ne se fait pas scrupule de participer au démantèlement des mythes nationaux. Son attitude n'est nullement à cet égard celle d'un conseiller politique. Nous avons vu d'ailleurs que, lorsqu'il souligne l'intérêt proprement politique de ceux qui gouvernent à reconnaître la »vérité«, il considère comme une chose allant de soi que ceux-ci ne sont nullement maîtres de décider du vrai et du faux sur ces matières. Cela suppose une forte conscience de soi de l'érudit comme appartenant à une caste qui s'est imposée désormais dans le champ social, à l'ombre des pouvoirs politiques et religieux. Elle ne possède pour autant aucune autonomie institutionnelle, comme le montre l'absence d'indépendance effective des institutions du savoir anciennes (universités...) et nouvelles (académies).

On voit cependant combien cette production collective d'un savoir démystificateur, qui se fait au nom des intérêts de la république des lettres, obéit à sa propre dynamique et à sa propre logique, et ne peut éviter d'entrer en conflit avec les intérêts politiques et religieux. Cette même lettre nous apprend que Naudé projette un ouvrage ambitieux, où toutes ces fables et mystifications seraient combattues »ensemble«, une sorte, pour ainsi dire, d'encyclopédie des impostures lettrées, politiques et religieuses. Il en évoque alors une, à titre d'exemple, qui lui tient à cœur, mais pour regretter aussitôt de ne pouvoir se permettre de mener à bien l'opération: »Il y a néanmoins celle des évêques de Ravenne créés par le St Esprit qui mériterait bien d'être traitée à part. Mais le pays où j'en suis m'en empêche, car que ne diraient-ils point si je leur montrais que c'était un pigeon de bois qui emittebatur ex machina quadam pour symbole du St Esprit, que l'on croyait présider invisiblement à cette élection « ${ }^{30}$. Exemple proprement paradigmatique de Deus ex machina, que ce Saint-Esprit littéralement sorti d'une machine dans le grand théâtre des impostures politico-religieuses: on comprend qu'il plaise particulièrement à Naudé, qui avoue cependant qu'une telle démystification est proprement impubliable en Italie.

\section{CONCLUSION PROVISOIRE}

Ce que Naudé considère, à juste titre, comme une information notable pour la république des lettres - la mise en évidence d'opérations frauduleuses des gersénistes pour confirmer leur candidat à l'auctorialité de l'»Imitation« - est pris dans un contexte politico-juridique qui en offre la condition de possibilité et lui confère son autorité. Sans doute Jacques Dupuy, proche de Naudé, n'avait-il guère besoin de telles assurances pour croire à son récit et tenir pour une chose avérée la fraude du bénédictin; mais si Naudé peut lui demander instamment et presque lui ordonner d'intervenir auprès de Richelieu, c'est que son expertise est validée par la présence d'une autorité ecclésiastique (Bagno) et de son notaire. C'est fort de ces circonstances et de ce document que Naudé pourra plus tard défendre son expertise dans ses pamphlets et devant les tribunaux. Mais on voit aussi par là que la république des lettres - c'est une plate évidence,

30 WolfE (éd.), Lettres de Gabriel Naudé (voir n. 2), p. 122. 
qui vaudrait tout aussi bien aujourd'hui - n'offre elle-même aucun cadre propre, aucune instance autonome permettant d'autoriser une expertise dénonçant la falsification dont l'un de ses membres au moins s'est rendu coupable. Le conflit, en outre, qui oppose des lettrés aux lettrés, est évidemment interne à la république des lettres (au sens où tous les protagonistes peuvent revendiquer en être des membres), mais ceux qui s'y trouvent aux prises y interviennent non pas seulement ni d'abord en tant qu'ils en sont citoyens, mais comme serviteurs d'institutions et de pouvoirs qui lui sont extérieurs: l'ordre, en ce qui concerne les bénédictins ou les chanoines réguliers de Saint-Augustin; quant à Naudé, il agit en faveur des intérêts du cardinal Bagno, dont il est le secrétaire et le bibliothécaire, en même temps qu'il montre sa fidélité à Richelieu. Il n'en est pas moins certain qu'il conçoit spontanément l'issue de son expertise comme intéressant immédiatement la république des lettres, en tant qu'elle s'intègre très exactement dans cette sorte de programme intellectuel auquel il ne cesse de travailler en sous-main: traquer, confondre et dénoncer, chaque fois que cela est possible, les opérations de falsification dans tous les domaines du savoir et en particulier dans celui des belles lettres, où il excelle par son érudition. Ses outils majeurs sont la science historique et la philologie, mais rien ne le sépare substantiellement, du point de vue de la démarche, des nouvelles orientations des sciences de la nature. Il y a une indéniable continuité et homogénéité entre, d'une part, l'analyse des impostures livresques, fondée sur l'érudition et l'examen direct, et d'autre part la critique, appuyée sur la raison et l'expérience, des prodiges ou phénomènes surnaturels, appréhendés euxmêmes comme des mensonges et des supercheries ${ }^{31}$. Il s'intéresse lui-même aux questions scientifiques - il a laissé un écrit sur l'éruption du Vésuve ${ }^{32}$ - et fréquente plusieurs des membres de l'académie des Lincei ${ }^{33}$. Dans les deux cas, il s'agit non seulement de décrire, mais aussi d'expliquer l'imposture en fonction de ses fins, à la fois particulières et toujours réductibles à l'étroite et indispensable intrication de la politique et de la religion. C'est là qu'interviennent toutes les limitations à la fois imposées et reconnues légitimes à la diffusion et à la publication d'un savoir de l'imposture, qui met nécessairement en question les pouvoirs et prérogatives fondés sur celle-ci (par exemple il ne peut-être innocent de publier que les prodiges et miracles tenus comme fondateurs de la monarchie française ne sont que des artifices ou des fables). C'est là aussi que cette science peut révéler toute son utilité politique; non pas dans l'émancipation populaire, mais dans la production, à l'usage de l'élite régnante, d'un

31 On renverra ici aux nombreux travaux sur la question d'Antony Grafton, Lisa jardine, Peter Dear, Ann Blair, Barbara Schapiro, etc. dont on trouve une bibliographie raisonnée, fort utile, dans Steven SHAPIN, The Scientific Revolution, Chicago 1996; ID., La révolution scientifique, trad. C. Larsonneur, Paris 1998, p. 228-229.

32 Gabriel NAudÉ, Discours sur les divers incendies du Mont Vesuve et particulièrement sur le dernier qui commença le 16 décembre 1631, s.l.n.d. [1632].

33 Un exemple parfait de cette continuité et homogénéité est fourni, tout près de Naudé, par JeanJacques Bouchard, grand érudit, peut-être plus radicalement »déniaisé« que Naudé ne l'était lui-même. Son »Journal d'Italie« montre en effet comment il adopte la même attitude intellectuelle dans ses travaux d'érudition, dans son insatiable poursuite des supercheries religieuses et dans son vif intérêt pour les sciences de la nature. 
savoir efficace de l'imposture; c'est-à-dire à la fois efficace pour se garder de l'imposture et pour créer de l'imposture. Il nous semble que c'est ce qu'accomplit l'ouvrage le plus fameux de Naudé, les »Considérations politiques sur les coups d'État«, écrit, si l'on en croit du moins la préface, pour l'instruction et le service du cardinal de Bagno, qui briguait le trône de saint Pierre. Mais il s'agit bien d'un savoir à deux tranchants, car il n'est efficace que parce qu'il est vrai, et il n'est vrai que parce qu'il confond l'imposture. C'est en tant que son savoir a pour fin la vérité, indépendamment de toute utilité sociale et politique, que le lettré revendique son appartenance à une république des lettres, mais au moment même où il s'emploie à faire connaître ce qu'il a appris en matière d'imposture et de falsification se pose tout à la fois la question de la censure nécessaire de ce savoir et de son utilité possible, aux dépens de la vérité, dans le cadre du service que le lettré est susceptible d'apporter aux pouvoirs qu'il sert: savoir secret de l'expert en imposture (l'aide à la réalisation des coups d'États les plus efficaces) et savoir de l'écrit au plus grand profit de ses maîtres (des pamphlets politiques par exemple). Certes, il est un fait entendu que la révélation publique de bien des vérités touchant à la religion et donc à la politique ne saurait être que dommageable pour l'ordre social; à la fois, dans l'optique même des combats politiques auxquels participe le lettré lorsqu'il y est requis, la dénonciation des impostures de l'adversaire peut être fort utile, et aucune borne sociale définitive ne saurait non plus limiter l'instruction »libertine« aux seuls pairs et aux seules élites politiques au service desquels travaille le lettré, qui ne peut pas ainsi, quelles que soient ses précautions (d'ailleurs toutes relatives) de confidentialité dans la publication, ne pas participer à la diffusion toujours plus large d'un esprit critique en matière de prodiges, miracles et impostures. Pour aller très vite: emporté, bon gré mal gré, dans une dynamique de publication, inséparable du médium de l'imprimé et des nouveaux canaux de diffusion des textes manuscrits et imprimés, le lettré libertin, pourtant contraint par les multiples dispositifs de censure et d'autocensure, ne peut pas ne pas œuvrer dans le sens d'une rationalisation globale des savoirs et des comportements, à rebours de ce qu'il lui semble percevoir des intérêts et nécessités politiques. Le syntagme de »république des lettres « au XVII ${ }^{\mathrm{e}}$ siècle est pris dans ce nœud de tensions et de contradictions. Projection d'une communauté idéale autonome où la »vérité« pourrait circuler à l'abri des persécutions et sans compromettre l'ordre social et politique, il est inséparable d'une conception éminemment aristocratique de la vie lettrée; dans le réel, un tel lieu social n'existe évidemment pas: les lettrés demeurent strictement inféodés aux pouvoirs et institutions auxquels ils sont attachés dans leur activité même de producteurs de savoir et de texte, ce qui crée nécessairement des conflits acharnés et impitoyables là où devraient régner amitié et tolérance, selon le code déontologique promu à travers le syntagme de »république des lettres«, mais ce service des grands et ces conflits internes ne sauraient avoir lieu sans que l'information ne circule et ne pénètre profondément le corps social tout entier, érigé en »public«, devenant ainsi, dans l'arbitrage des conflits et la discussion autour de leurs enjeux politiques, partie prenante de cette »république« virtuelle, où, a priori, aucune place ne lui était réservé. 


\section{CODA 1 \\ LE COMBAT DE NAUDÉ CONTRE LES BÉNÉDICTINS ${ }^{34}$}

L'affaire prend une nouvelle dimension, publique et juridique, après la publication par le chanoine régulier de Sainte-Geneviève, Jean Fronteau, à la suite de son édition de l'»Imitatio « parue en 1649 sous le nom de Kempis, d'une longue réfutation latine des thèses gersénistes de Gaetani (»Thomas a Kempis Vindicatus«) contenant une lettre latine de Naudé à Fronteau (16 avril 1647) - où l'érudit laisse éclater son hostilité à l'égard de l'abbé bénédictin - ainsi que la copie du rapport d'expertise tirée des archives des frères Dupuy 35 . Il faudrait reprendre et poursuivre les analyses précédentes à la lumière des nombreuses péripéties éditoriales et juridiques qui, à notre connaissance, n'ont à ce jour fait l'objet d'aucune étude approfondie. Il manque même le récit circonstancié des événements. Faute d'être en mesure de traiter du dossier de manière exhaustive, nous donnerons cependant les grandes lignes de la querelle, car elle présente de nombreux aspects faisant apparaître, nous semble-t-il, combien le processus d'autonomie du champ lettré était encore à cette époque balbutiant.

En tout état de cause, Naudé semble avoir pris l'initiative de l'offensive, car il ne se contenta pas d'assister Fronteau dans sa polémique par sa lettre et son rapport, mais publia la même année dans son »Mascurat" plusieurs pages extrêmement violentes contre Gaetani, sans le nommer, mais en le désignant de manière transparente. Celui-ci en effet avait écrit quelque part que le père de Mazarin, sicilien comme lui, n'était qu'un simple »chapelier« et »boutonnier« ayant fait banqueroute. Une vraie aubaine pour Naudé, qui utilise la couverture politique d'une réparation des torts procurés au nom de Mazarin pour attaquer l'abbé, dans le style le plus satirique, le traitant de »moine crotté, rabougri, ratatiné, fol et enragé à mesdire de tout le monde« et dénonçant ses multiples »impostures«, dont, évidemment l'invention de Gersen ${ }^{36}$.

34 Je tiens ici à remercier tout particulièrement Fabienne QUEYROUX, qui m'a permis de consulter sa thèse à paraître, Recherches sur Gabriel Naudé, doctorat de l'École nationale des chartes, 1990. Ce travail contient un chapitre entièrement consacré à la querelle de l'»Imitation « (chap. VII) et en retrace le déroulement avec plus de précisions et de détails. En outre les 13 lettres de Naudé au chanoine génovéfain Boulart conservées à la bibliothèque Sainte-Geneviève s'y trouvent reproduites et annotées en annexe.

35 Thomæ a Kempis canonici regularis ordinis S. Augustini De imitatione Christi libri quatuor. Ex recensione P. Joannis Frontonis, canonici regularis Stae Genovefae, ordinis S. Augustini. Cum evictione fraudis qua nonnulli usi, id operis cuidam Joanni Gersen ascripere, Parisiis, apud Sebastianum Cramoisy [...] et Gabrielem Cramoisy, 1649. Le rapport d'expertise est présenté p. 12-14, avec la mention: »ex volumine 588 chartularij nobilissimorum Fratrum Puteanorum«. Voir en annexe la traduction du texte présentée par Jean Fronteau en 1652. La description que nous tentons ci-dessous est minimale (on se reportera à la thèse de F. Queyroux, voir $n$. 34) et surtout myope. Il faudrait évidemment s'employer à insérer les épisodes successifs de la querelle dans le contexte politique de la Fronde, Naudé, comme on le sait, s'étant illustré par son soutien indéfectible à Mazarin son patron. L'essentiel du travail reste donc à faire.

36 »Pourquoy ce maistre Moine, ou Abbé racoucy de Trastevere, auroit-il esté plus heureux en cette imposture, qu'en beaucoup d'autres par luy effrontément publiées sur Gregoire le grand, sur Gelase second, sur S. Ignace, sur Paul IV, sur Thomas a Kempis, \& nouvellement encore sur les Peintures de l'Eglise de S. Sebastien de Rome, esquelles il a changé la Vierge en Saintc 
La riposte ne se fait guère attendre. Elle vient d'un bénédictin de l'abbaye de SaintGermain-des-Prés, de la congrégation de Saint-Maur, Robert Quatremaires, et d'un bénédictin anglais du couvent de Cluny, déjà engagé dans la controverse au service de Gaetani, Francis Valgrave (Anthony Timcock), lesquels, en deux ouvrages dirigés d'abord contre le père Fronteau, s'indignent de la diffamation et des injures contre le très respecté abbé romain (qui décède d'ailleurs l'année de la parution de l'ouvrage). Mais plus encore, Naudé est accusé d'avoir établi un faux rapport et falsifié lui-même les manuscrits ${ }^{37}$. De plus, selon les deux religieux, son témoignage serait éminemment partisan, car il est titulaire du prieuré commendataire de l'Artige, qui relève de l'ordre

Benoist, \& les Saincts Marc \& Marcellin en Sainct Pierre \& Sainct Paul, y adjoustant des inscriptions à sa mode; \& sur une infinité d'autres, lesquelles ont donné sujet à ces brillantes lumieres de l'Academie Romaine Messieurs Lucas Holstenius, Leo Allatius, Janus Nicius Erythraeus, au R P Ro de la Compagnie de Jesus, au sieur Naudé, \& auparavant eux encore à Antonius Gallonius, \& à Joan. Baptista Castaldus: \& finalement à tous ceux qui le connoissent, $\&$ qui ont tant soit peu conscience \& de probité, de le publier pour le plus grand fourbe $\&$ imposteur qui soit en Italie. Praestigiator ille, nam quo alio cum nomine appellem nescio, ce sont les paroles de Janus Nicius en l'epistre rapportée par le sieur Leone Allatio, qui suis quasi cantionibus homines immutare, atque aliam illis formam \& imaginem dare conatur, ille inquam, qui Ignatium virum sanctissimum, notissimumque in Eneconem nescio quem vertit, proximo superiore anno, novis ut ita dicam cantionibus, non magis impudenter quàm stultè, fucum est nobis facere conatus, ac rem unam pro alia obtrudere [De Occid. \& Orient. Eccles. Perpet. Consensione lib. 1. cap. 6. p. 131] Et apres ces tesmoignages si authentiques, contre ce faussaire, pourra-t'on adjouster foy à cette belle metamorphose qu'il luy a pleu faire, d'un Gentilhomme bien né, \& assez accommodé des biens de fortune, pour se contenter de la part que Dieu luy en avoit faite, un un pauvre marchand Chapelier, Boutonnier, \& qui plus est Banqueroutier, comme il a fait du pere du Cardinal. Mais quoy il se vouloit peut estre vanger de quelque differend qu'il avoit eu avec le Pere Jules Mazarin Jesuite touchant la fondation de certains Colleges en Sicile, puisque ledit Pere au rapport d'Alegambe, Studia quaedam erexit in Sicilia, où cet imposteur avoit pareillement dessein d'en establir pour quelques Religieux de son Ordre; ou bien parce qu'il avoit trouvé quelque Matarin, ou Mascarin, ou Marcasin, aux environs de Palerme, qu'il s'est imaginé de pouvoir faire passer pour Mazarin, ne plus ne moins qu'il changea il y a plus de vingt-cinq ans Gerson en Gersen, afin de faire croire au monde que les livres de Imitatione Christi de Thomas à Kempis publiez anciennement je ne sçay quelle inadvertence, sous le nom de ce fameux Chancelier de Paris Jean Gerson, avoient esté faits par un Abbé de l'Ordre de S. Benoist nommé Jean Gersen. Et certes puis que Gregoire XIII. comme a fort bien remarqué ce dernier Honneur de la Grece Leone Allatio, en la seconde edition de la Censure contre les Antiquitez de Volterre, ou plustost de ces Fragmenta Etrusca, publiez par le sieur Inghiramo, fit punir de mort un certain Alfonso Ciracarelli da Bevagna, parce qu'il fut convaincu d'avoir commis beaucoup de faussetez en matieres d'Histoires \& de Genealogies: je ne sçay pourquoi l'on n'empesche point à Rome ce Moine Crotté, d'en publier tous les jours de si remarquables, tant en l'Histoire Ecclesiastique, que profane ou civile, Jugement [...]«, Vienne, Bibliothèque d'État, ms 7071, p. 46-48. Sur l'appui qu'il obtient de ses amis italiens, voir infra.

37 Joannes Gersen, abbas Vercel. ord. S. Benedicti, auctor libb. de Imit. Christi iterum assertus, a Domno Roberto Quatremaires, [...] contra refutationem P. Joan. Fronteau, Parisiis: apud J. Billaine, 1650; Argumentum chronologicum contra Kempensem, quo Thomam a Kempis non fuisse, nec esse potuisse authorem librorum de Imitatione Christi, adversus Joannis Frontonis, [...] (Thomam a Kempis vindicatum) demonstratur per Franciscum Valgravium [...], Parisiis: sumptibus J. Billaine, 1650. 
des chanoines de Saint-Augustin (en oubliant de préciser que ce n'était pas le cas au moment de l'expertise). C'est alors de façon apparente que Naudé, le 17 août 1650, porte l'affaire sur le terrain juridique, en demandant la censure des écrits des bénédictins devant le préfet de Paris, obtenant le séquestre des ouvrages de Robert Quatremaires et de François Valgrave.

Parallèlement, il tente d'obtenir une »conférence« en présence de toutes les parties et d'arbitres désignés, sur le modèle de celle qui avait opposé Ramus et Charpentier sous l'autorité du cardinal de Lorraine (1565), et, plus récemment, Richelieu n'avait pas hésité à réunir une commission pour juger de la valeur de la méthode de Morin pour calculer les longitudes ${ }^{38}$. Cette »conférence« n'aura jamais lieu.

En revanche, les bénédictins contre-attaquent, sur le terrain juridique comme sur celui de la controverse. Ils parviennent à faire lever la saisie, poursuivent à leur tour Naudé et publient un factum, dans lequel, entre autres accusations, ils reviennent sur les invectives du »Mascurat « lancées contre Gaetani et en particulier sur le mot »rabougri«, injure qui à elle seule »merite punition exemplaire«, si l'on veut bien y voir une allusion à »bougre « (sodomite) ${ }^{39}$. Naudé ne niera pas avoir traité l'abbé de »rabougri« (le »Mascurat« avait été publié sans nom d'auteur, mais l'attribution ne faisait aucun doute) et retournera même l'injure, non sans provocation, contre Quatremaires ${ }^{40}$, mais il prendra cette accusation d'avoir voulu suggérer que le bon père faussaire était de plus sodomite suffisamment au sérieux pour se justifier et disserter longuement sur le sens du terme et son étymologie et surtout pour solliciter une consultation officielle de l'Académie française sur le sens du terme. Ainsi publiera-t-il, dans un factum de 1651, le »sentiment « de l'Académie, par l'entremise de deux signatures amies ( $\mathrm{La}$ Mothe le Vayer et Colletet), où il apparaît que le terme »rabougri« désigne une malformation physique et nullement un vice ou une faute morale, faisant ainsi un usage à la fois juridique et polémique de cette très officielle et artificieuse attestation ${ }^{41}$.

38 Voir la lettre de Naudé du 24 novembre 1650 au chanoine génovéfain Boulart, transcrite et annotée par F. Queyroux, dans sa thèse (voir n. 34).

39 Factum pour dom Placide Roussel, prieur de l'abbaye Saint-Germain-des-Prés, ordre S. Benoît, congrégation S. Maur, et dom Robert Quatremaire, religieux de ladite abbaye, défendeurs, les religieux bénédictins de ladite congrégation S. Maur intervenants et incidemment demandeurs, contre maître Gabriel Naudé, médecin et prieur commendataire de l'Artige, ordre de S. Augustin, demandeur et défendeur, s. 1. 1651.

$40 »$ le bon P. Quatremaire, qui est si Rabougri_luy-mesme«, Raisons péremptoires [...], ibid.

41 »vous sçaurez que Monsieur Conrart proposa hier à Messieurs de l'Academie vostre mot Rabougry, pour sçavoir la vraye \& naïve signification. Quoy que la Compagnie fust alors fort grande, si est ce qu'il n'y eut point en cela de divers advis. Car tous nos Messieurs demeurent unanimement d'accord, que ce mot ne signifioit rien autre chose qu'un corps imparfait, entassé $\&$ racourcy. Et pour le justifier encore, on rapporta quelques articles des Ordonnances sur le sujet des forest, où il est fait mention de certains arbres, qui y sont nommez Rabougris, pour dire, qu'ils n'ont pas toute leur juste croissance. J'adjousteay que l'on disoit aussi un fruit rabougry, une poire ou une pomme rabougrie, pour dire tantost vieille \& ridée, \& tantost avortée, qui n'a ny toute sa consistence, ny toute sa maturité requise; ce qui me fait croire que ce mot pourroit biend eriver du mot Latin Abortius, \& c'est aussi le sentiment de quelques uns de nos plus doctes amis. Quoy qu'il en soit, il est certain, \& la Compagnie l'a conclu tout d'une voix, 
Il est très important de souligner que Naudé n'agit pas seul, mais en constante liaison avec les chanoines de Sainte-Geneviève, où il réside d'ailleurs. On peut même dire que, au plus fort de la polémique, Naudé, loin d'apparaître comme un héros solitaire de la république des lettres qui récuse les positions partisanes, sert les intérêts moraux de l'ordre auquel Kempis appartenait. Par son intervention, on peut dire qu'il soutient et seconde les efforts du génovéfain Fronteau. Du reste, en juillet 1651 les chanoines de Sainte-Geneviève s'engagent eux-mêmes dans la bataille juridique, comme "parties intervenantes en la cause«, et les chanoines réguliers de Saint-Victor se joignent à eux au début de l'année suivante. Par ailleurs le chanoine Boulart, avec lequel Naudé est en contact permanent, rédige une circulaire destinée à tous les monastères de l'ordre afin de collecter des manuscrits de l'»Imitatio« où le nom de Kempis apparaît ${ }^{42}$.

Sur le terrain de la controverse, Naudé se montre d'une activité proprement frénétique. Il sollicite l'aide de ses anciens amis et protecteurs italiens - Leo Allaccio, Lucas Holstein, Cassiano Dal Pozzo, Francesco Barberini lui-même - pour attester sa bonne foi et la raison de sa cause, obtient des textes de trois d'entre eux, qu'il fait publier ${ }^{43}$. Il parvient ainsi à mobiliser des membres éminents de la république des lettres, ou plus exactement les membres de son propre réseau romain, mais leur apport est intégré à un ensemble où l'action politico-juridique est première et déterminante, et où Naudé est devenu, de fait, comme on l'a dit, un auteur au service des génovéfains. Ceux-ci, du reste, se montrent d'une vitalité polémique remarquable, à travers une série de publications en latin et en français ${ }^{44}$. Mais Naudé travaille tout autant à se ménager tous les

que ce mot ne designoit aucune corruption, ny depravation de moeurs, \& n'eut jamais ce sens obscene que quelques-uns luy veulent donner«. Cette missive, datée du 4 janvier, est signée du nom de Colletet. Mais elle ne satisfit pas tout à fait Naudé, s'il est vrai qu'il revient à la charge le 17 février par le mot suivant destiné à Le Vayer, qui lui fera une réponse tout à fait similaire à celle de Colletet: »L'Autheur d'un Livre du temps, s'estant servy du mot de Rabougry, en parlant d'un homme qui estoit petit et mal-fait, il se trouve que quelques siens amis l'ont pris pour une injure, comme si on l'avoit voulu taxer d'un crime en ses moeurs, à quoy il y a bien de l'apparence que l'on n'a point pensé. Et pour ce qu'en mon particulier, je croy aussi que cette parole n'a jamais esté prise en un si mauvais sens, je vous prie de sçavoir determinément de Messieurs de l'Academie Françoise, ausquel j'ay sceu que Monsieur Conrart en avoit desia parlé, quelle est sa vraye signification, \& si elle a esté quelquefois employée en si mauvaise part«. Naudé publie ces textes dans son »Advis publié pour la seconde fois sur le factum des PP. Dom Placide Roussel, Prieur de l'Abbaye S. Germain des Prez, Dom Robert Quatremaire, Religieux deladite Abbaye. Et les Religieux Benedictins de la Congregation de sainct Maur, intervenans. Contre Maistre Gabriel Naudé«, qui se trouve dans: Placet imprimé des PP. bénédictins, demandeurs en fait de mainlevée, contre maître Gabriel Naudé, défendeur, avec les réponses et corrections dudit Naudé, [...] ensemble un Avis sur le factum desdits pères bénédictins, s.1. 1651, p. 22-24.

42 Cette correspondance (éditée par F. Queyroux), le texte de la circulaire et de nombreuses autres pièces se trouvent à la bibliothèque Sainte-Geneviève, ms 289 et 290.

43 Testimonium adversus Gersenistas triplex: Lucae Holstenii, Leonis Allatii, Camilli de Capua, [...] ab Anton. Franc. Payen, [...] literis consignatum, Parisiis: S. et G. Cramoisy, 1652.

44 Outre ceux de Jean Fronteau, voir les ouvrages des pères G. de Boissy et Desnos. En particulier: Jean FronteAU, La Contestation touchant l'auteur de l'Imitation de Jésus-Christ rendue manifeste par l'opposition de toutes les preuves proposées par les bénédictins et les chanoines 
soutiens possibles hors des génovéfains et intervient dans trois ouvrages kempistes au moins produits par des ecclésiastiques d'horizons divers ${ }^{45}$. Il publie lui-même, outre le factum déjà cité, un ouvrage substantiel en latin entièrement consacré à l'affaire mais, comme tous ses autres ouvrages, plein de particularités érudites ${ }^{46}$ - et un pamphlet en français reprenant, résumant et précisant les arguments avancés dans le précédent ${ }^{47}$. Naudé, évidemment, y réfute point par point les accusations formulées par les bénédictins à son encontre, mais surtout demande que l'on refasse l'expertise des manuscrits, requête que ses adversaires ne cessent d'éluder. Il se propose lui-même »de consigner douze cents livres, pour estre converties au profit de l'Hostel Dieu, au cas que les Benedictins luy permissent de faire venir ces MSS et qu'ils ne fussent

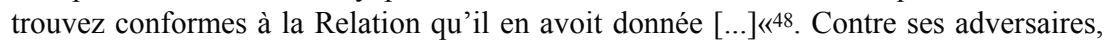
Naudé ne désarme pas, la polémique devenant même une mise en cause de l'ordre de Saint-Benoît comme tel, s'il est vrai qu'il dresse une très longue liste, extraordinairement documentée, à son habitude, de tous les faux - faux privilèges, indults, octrois, fausses lettres, etc. - produits par les religieux bénédictins depuis les temps les plus anciens. Ainsi les impostures et les falsifications de Gaetani ne sont-elles pas une déplorable exception, mais la règle même, si l'on peut dire, de l'ordre bénédictin depuis sa fondation.

On pourrait avoir l'impression, à lire ces pages téméraires, que Naudé cette fois va trop loin. Il finit pourtant par obtenir satisfaction sur l'essentiel: par un décret du 12 février 1652, le parlement de Paris »fait defense d'imprimer le livre De Imitatione Christi sous le nom de Jean Gersen, abbé de Verceil; permet audit abbé et aux religieux de SainteGeneviève et de Saint-Victor de continuer à les faire imprimer au nom de Thomas a Kempis, et défend aux parties de plus récidiver, sans dépens $\aleph^{49}$. Ainsi la querelle, loin

réguliers, divisée en trois parties, avec les preuves justificatives du droit de Thomas de Kempis, Paris: S. et G. Cramoisy, 1652.

45 Thomas CARRE [Miles Pinkney], Thomas de Kempis a seipsa restitutus, Paris, Vidua H. Blageart, 1651; Theophilus EuSTATHIUS [très probablement Michel Constantin], Argumenta duo nova, primum Theophili Eustathii P. T. [...] Alterum J. Frontonis [...] quibus demonstratur adversus Pseudo-Gersenistas Thomas Kempensem verum esse auctorem librorum de Imitatione Christi, Paris, Cramoisy 1651; Georgius HESER, S. J., Adversus pseudo-Gersenistas praemonitio nova, Paris 1651. Sur cette intense activité éditoriale, voir Paul Oscar KRISTELLER, Between the Italian Renaissance and the French Enlightenment: Gabriel Naudé as an Editor, dans: Renaissance Quarterly 31/1 (1979), p. 41-71.

46 Gabriel NAUdÉ, Causae Kempensis conjectio pro curia romana a Gabriele Naudaeo actore et sodales quosdam Benedictinos quinque falsitatum arcessente, scripta [...], Paris 1651.

47 ID., Raisons peremptoires de maître Gabriel Naudé [...] contre D. Placide Roussel, Robert Quatremaire et François Valgrave, religieux bénédictins defendeurs, \& demandeurs en mainlevée des livres sur eux saisis. Et les Congregations de Sainct Maur \& de Clugny intervenantes. Pour montrer que les IV Mss. de Rome dont les dits bénédictins se servent pour ôter le livre de l'Imitation de Jésus-Christ à Thomas de Kempis et le donner à un supposé Gersen, sont falsifiez et qu'ils ne peuvent l'avoir esté que par le nommé Constantin Cajetan, religieux bénédictin, ou par quelque autre du mesme ordre [...] s.l.n.d. [1651].

48 Ibid., p. 13.

49 Jugement contradictoire de nosseigneurs des Requêtes du Palais du parlement de Paris, après six audiences, sur la contestation mue entre les chanoines réguliers de S. Augustin de la 
de se régler sur le terrain des lettres, est-elle tranchée par décision de justice: le parlement de Paris intervenant pour interdire la publication de l'»Imitation « sous le nom de Gersen, au profit de celui de Kempis. Un critique moderne, gerséniste acharné, a beau jeu de remarquer combien »ces magistrats se montrèrent plutôt présomptueux en s'arrogeant le droit de légiférer dans une question storico-littéraire; quelle valeur pouvait avoir leur verdict dans la république des lettres? «50 En effet, la querelle n'était pas réglée pour autant, et Naudé lui-même eut le temps d'écrire avant de mourir, l'année suivante, une réfutation d'un ouvrage de Jean de Launoy dirigé contre les arguments kempistes ${ }^{51}$.

\section{CODA 2 \\ UNE QUERELLE INDÉFINIMENT RECONDUITE}

Mais surtout, chose notable dans les annales de la république des lettres, la querelle sur l'attribution de l'»Imitation" se poursuit aujourd'hui encore et en des termes, comme le remarque un auteur, tout à fait similaires à ceux du XVII ${ }^{\mathrm{e}}$ siècle, concernant en tout cas la distribution des camps, puisque kempistes, gersonistes et gersenistes sont toujours en lice ${ }^{52}$. Il est frappant de constater comment, dans les travaux les plus impartiaux, les arguments de fond contre l'attribution à Gersen (étrangement soutenue par, entre autres défenseurs, un... chanoine régulier de Saint-Augustin ${ }^{53}$ ) restent ceux-là mêmes qui étaient avancés par Naudé, mais interprétés a minima. On veut dire par là qu'ils se ramènent tous en substance à des erreurs de copie et de lecture du nom de Gerson, sans qu'ils s'aventurent jusqu'à suggérer d'éventuelles malversations ${ }^{54}$. Mais

congrégation de France, et les religieux bénédictins de la congrégation de S. Maur, portant que les livres de l'Imitation de Jésus-Christ seront dorénavant imprimés au nom de Thomas à Kempis, chanoine régulier de S. Augustin, et que défenses sont faites de les plus imprimer sous celui d'un supposé Jean Gersen, prétendu bénédictin. Du 12 février 1652, factum, 1652.

50 Tiburzio LuPO, L'Imitazione di Cristo e il suo autore [...], vol. 1, Torino 1964, p. 137 (nous traduisons).

51 Réponse aux remarques sommaires du sieur Jean de Launoy. [...] touchant l'auteur des IV livres de l'Im., adressé à l'auteur de la Contestation rendue manifeste, chanoine régulier de Saint-Augustin, par Gabriel Naudé, bibliothécaire de la sérénissime reine de Suède, Paris 1653.

52 Voir, pour la thèse gersoniste, toujours défendue par les gallicans: Examen d'une question célèbre qu'a fait naître l'humilité de Gerson, et qui, pendant deux siècles, a partagé le monde savant, Le Gallican, juillet 1983 http://www.gallican.org/gerson4.htm (29/03/10). Un nouveau candidat, mais défendu avec une beaucoup plus grande prudence philologique, est apparu: Conradum de Fritzlaria (seconde moitié du XIV ${ }^{\mathrm{e}}$ siècle).

53 Brian MCNEIL, De »L'Imitation de Jésus-Christ«, traduit de l'anglais par Éliane Utudjian Saint-André, Paris 2002. Ses arguments, d'une rare indigence philologique, sont contestés dans une postface par G. Epiney-Burgard, plutôt kempiste, qui remarque: »régulièrement, on projette de reprendre à zéro l'étude des manuscrits et de leurs variantes, ce qui serait aujourd'hui facilité par l'usage de l'ordinateur«, p. 137. Mais l'ordinateur ne fait pas tout; il faudrait peutêtre y ajouter un esprit critique à la Naudé ou à la Bayle!

54 Voir la notice de B. SPAAPEN, »Imitatio Christi«, dans: Dictionnaire de spiritualité, t. VII, col. 2338-2363; et Albert AMPE, S. J., L'»Imitation de Jésus-Christ« et son auteur, Rome 1973. 
aussi peut-on constater que l'expertise de Naudé n'est, dans aucun des travaux, prise au sérieux ni véritablement en considération, même si, pour un des documents au moins, elle est corroborée par la critique55. L'accusation surtout de falsification de la part de Gaetani se trouve rejetée a priori, sans aucune justification. La notice récente consacrée à l'abbé dans la »Biografia degli Italiani« dit seulement: »Naudé tenta même de certifier les falsifications de Gaetani mais la critique moderne ne donne aucun crédit à cette initiative ${ }^{56}$. Le bibliophile semble en outre s'être discrédité par les propos satiriques du »Mascurat «: »Une harangère en colère serait un exemple de modération en comparaison de ce M. Naudé«, disait déjà le dominicain Thuillier en 1724, repris par $\mathrm{M}^{\mathrm{gr}}$ Puyol ${ }^{57}$ : »Mais aussi, dit celui-ci, qu'allait-il se jeter en une aventure semblable? Surtout pourquoi tant d'impétuosité, d'irréflexion et d'étourderie? Lui, un avisé, un politique, un raffiné, il prenait couleur, sans nécessité, sans intérêt, en une question de corps; et il intervenait tout à la fois avec rudesse et légèreté dans une querelle où était engagé l'amour-propre littéraire et national!«58 Outre le fait que ces textes montrent combien les modes d'expression dans les querelles lettrées ont changé depuis l'époque de la Fronde, jusqu'à rendre incompréhensible la verve satirique de Naudé, on voit aussi que l'idéal d'une république des lettres résolument critique est encore loin d'être partagé par un professeur de Sorbonne (certes, engagé dans les prélatures) au tournant de 1900. Certains vont plus loin encore, discréditant la figure morale de Naudé. Ainsi, en 1964, le salésien gerséniste Tiburzio Lupo, celui-là même qui s'offusquait de l'intrusion abusive du parlement de Paris sur les terres de la république des lettres, fait-il dire à Sainte-Beuve que le bibliophile était »un homme abominable sous tous les aspects«, et que son expertise est donc indigne d'une quelconque confiance $^{59}$. Évidemment, Sainte-Beuve, qui composa un fort beau portrait littéraire de Naudé, ne dit jamais rien de tel; mais dans cette histoire nous n'en sommes pas à une imposture près!

55 À propos des ajouts manuscrits sur l'exemplaire de l'édition de L'»Imitatio« vénitienne (exemplaire dit »Genuensis«): ce qui acheva de discréditer cette note qui mentionnait le nom de Joannes »ce fut le témoignage, non pas de Naudé; il était suspect [sic!], mais d'Ughelli, de Wadding et de Victorelli, qui déclarèrent que l'inscription portait bien le mot de Joannes, mais que ce mot avait été grossièrement substitué à celui de Thomas, que l'on pouvait encore lire distinctement sous la surcharge«, Pierre Édouard PUYOL, L'auteur du livre »De imitatione Christi«, Paris 1900, $1^{\text {re }}$ section: La contestation, t. I, p. 225. F. Queyroux, conservateur à la bibliothèque de l'Institut de France, après avoir examiné les trois manuscrits incriminés (aujourd'hui conservés à Paris, Bibliothèque nationale, lat. 13603, 13599 et 13605), a pu constater l'exactitude et la précision de la description de Naudé.

56 M. CERESA, notice »Constantino Gaetani«, dans: Dizionario biografico degli italiani, vol. LI, p. 191.

57 Vincent Thuillier, Histoire de la contestation sur l'auteur du livre de l'Imitation de J.-C., Paris 1724, p. 19, cité dans PUYOL, L'auteur du livre »De imitatione Christi« (voir n. 55), p. 113.

58 Ibid.

${ }^{59}$ LuPO, L’Imitazione di Cristo (voir n. 50), p. 336. 


\begin{abstract}
ANNEXE
Copie du rapport d'expertise de Naudé envoyé aux frères Dupuy, traduit du latin par Jean Fronteau, La Contestation touchant l'auteur de l'Imitation de Jésus-Christ rendue manifeste par l'opposition de toutes les preuves proposées par les bénédictins et les chanoines réguliers, divisée en trois parties, avec les preuves justificatives du droit de Thomas de Kempis, Paris, S. et G. Cramoisy, 1652.
\end{abstract}

[p. 155]

\title{
RELATION DE L'EXAMEN FAIT À ROME DE QUATRE MANUSCRITS DONT ON VOULOIT SE PREVALOIR POUR JEAN GERSEN
}

\section{À la posterité.}

Les Peres Benedictins ayant eu advis que le livre de l'Imitation s'imprimoit à Paris dans le Louvre aux despens du Roy, de grand papier \& d'une lettre plus grande que les ordinaires, \& vrayement digne de sa Majesté, furent trouver l'Eminentissime Cardinal de Richelieu, \& le prierent de ne pas permettre que dans l'impression de cette excellente œuvre, on donnast lieu à l'erreur par lequel on l'avoit vulgairement attribué à Thomas de Kempis, veu qu'il appartient plustost à Jean Gersen Abbé de Vercelles de l'ordre de sainct Benoist, comme il apparoist plus clair que le jour tant des livres que l'Abbé Constantin a donné au public touchant cette matiere, que de la foy de plusieurs Manuscrits, dont la plus grande partie estoit gardée à Rome en la maison du mesme Abbé Constantin Caëtan avec le titre veritable \& non falsifié de Jean Gersen, \& non pas de Thomas de Kempis. L'Eminentissime Cardinal ne refusa point d'octroyer leur demande pourveu que les Benedictins de Rome representassent ces Manuscrits à quelque personne recommendable en dignité ou en doctrine, qui pust attester [156] avoir veu ces Manuscrits, les avoir diligemment examinez, \& qu'on les jugeoit tels qu'on pouvoit certainement s'y asseurer. À ce sujet le P. Placide, pour deuëment s'acquiter de la charge qui luy avoit été donnée, vint trouver 1'Eminentissime Cardinal de Bagny le 30 du mois de Janvier 1641 duquel ayant facilement obtenu que toute cette affaire se passeroit en sa presence, le lendemain 31 du mesme mois \& an, environ sur les 16 heures, le mesme P. Placide retourna, \& avec luy estoit le P. Jean, tous deux Procureurs de l'ordre de sainct Benoist de la Congregation de sainct Maur, \& apporterent quatre livres, lesquels moy Gabriel Naudé domestique dudit Cardinal, \& son perpetuel Commensal, je monstray audit Eminentissime afin qu'il en portast jugement suivant l'experience qu'il avoit acquise dans les Archives tant à Avignon qu'à Riette, du discernement du temps \& de la difference des plus anciens caracteres. Mais il jugea plus à propos qu'on prist deux Juges experts, qui en sa presence examineroient exactement cesdits livres, \& qui en diroient leurs advis, qu'un Notaire public recevroit en sa presence \& de quelques autres témoins. C'est pourquoy ayant été choisi sans l'avoir recherché par lesdits Peres pour l'un des Juges, \& ayant fait en sorte par ensemble que Floravante Martinelli tinst la place du second, je conduisis lesdits Peres au logis 
d'Adrien Gallus Notaire tres connû en la ruë dei Banchi, \& je luy donnay ordre de la part de mondit Seigneur le Cardinal de Bagny, pour commencer ce qu'il devoit faire après midy en presence dudit Cardinal, de reduire en forme d'instrument public, la description de ces quatre volumes, laquelle le [157] Pere Jean avoit apportée, recueillie ou par luy, ou par un autre avec beaucoup de diligence \& tres-conforme à la verité, \& ensemble les noms des Juges choisis, afin que incontinant après disner on pust plus facilement achever cette perquisition devant le susdit Eminentissime. Ces choses s'estant ainsi passées, m'estant ensuite retiré seul en ma chambre je commençay, avec un grand desir de satisfaire à ma conscience, de feüilleter lesdits livres, \& considerant tout de bien près, je trouvay que le livre du sieur Leone Allatio (c'est le Manuscrit qu'ils appellent Biscianus ou Romanus ${ }^{60}$ ) portoit à la verité le titre de Joannis de Canabaco, mais en telle maniere, que le mot de Canabaco paroissoit comme ômis par l'Ecrivain, hors de la ligne, \& un peu au dessus. D'autant neantmoins que cette omission pouvoit venir de l'escrivain, d'ailleurs estant de mesme main, escrite \& couchée de mesme couleur; sans m'arrester à cette difficulté, je feüilletay \& vis diligemment l'un après l'autre tous les traitez qui estoient dans le mesme volume, afin que s'il y avoit quelque datte je la pûsse trouver, \& de là tirer quelque conjecture du temps qu'a été écrit le livre de l'Imitation. Je rencontray donc une Bulle d'une certain Legat Apostolique en Allemagne, décrite en un seul feüillet de parchemin, \& donnée à Vienne l'an 1448. Ce qui me fit conjecturer, l'escriture de la Bulle estant la mesme que celle du livre de l'Imitation, que ce livret ne pouvoit en aucune façon avoir été écrit avant l'année 1448. Mais plutost beaucoup après, la Bulle ayant pû estre copiée seulement en 1480 ou au plus tard en 1500 pour ce que l'estat de cette escriture à peine permet-il qu'on la doive [158] juger plus ancienne que ce temps. Ensuitte ayant pris entre mes mains le second Manuscrit, (C'est celuy qu'il appellent de Mantouë61) je consideray premierement en son titre une inversion de paroles \& un ordre tout à rebours, en cette maniere: Incipit liber Ionannis Gersen primus, incontinant j'apperceus des effaceures d'un titre precedent \& plus ancien, duquel le nouveau suradjousté n'avoit pû bien couvrir certaines traces, comme, $I H O, \&$ quelques moitiez d'autres lettres, car elle paroissoient aussi tost qu'on les regardoit avec un peu d'attention. Et l'on ne pouvoit pas dire qu'elles fussent des traits des lettres qui estoient à l'opposite au revers du feüillet, pource qu'après Monsieur Floravante \& les mesmes Peres, les perçant par la pointe d'un ganif, reconnurent qu'il y avoit de la diversité. Enfin ces paroles liber Ionnis Gersen primus, estoient écrites d'un rouge beaucoup plus reluisant que les suivantes, de contemptu mundi \& Imitatione Christi: En sorte que de cette seule difference du rouge ou du vermillon, la fraude pouvoit estre manifeste à un aveugle, pour ne pas dire qu'il se trouve plusieurs autres marques en ce titre qui le font aussitost soupçonner de fraude \& de mensonge. C'est pourquoy bien que la Congregation de saincte Justine ait été en oubly depuis plus de deux cent ans, il est certain que cela ne donne aucune antiquité à ce titre, puiqu'il est tout à fait faux, \& sur-adjousté à un autre

60 Codex Allatianus, Paris, BN, fonds Latin 13605.
61 Codex Mantuanus, Paris, BN, fonds Latin 13603. 
plus ancien, quoy que pareillement faux, sçavoir Ioannis Gerson, à cause de ces trois vieilles lettres $I H O$, non pas, par un simple changement de Gerson en Gersen, mais par un renversement \& changement de [159] tout le titre: de peur que le mot Cancellarij estant laissé, ou quelque autre qui ne pouvoit pas si bien convenir à Gersen, il ne fist naistre l'occasion de découvrir la fraude: Car autrement la lettre, $\mathrm{O}$, du nom de Gerson, pouvoit tres-commodement estre changée en lettre, E, qui fait Gersen, de la mesme sorte qu'il est arrivé sur la fin de ce manuscrit en ces paroles, Explicit liber quartus Ioannis Gersen de sacramento Eucharistiae: Car je ne fais aucun doute qu'il y avoit auparavant Ioannis Gerson. Mais crainte que cette derniere inscription ne donnast lieu de connoistre la fraude de la premiere, elles ont souffert toutes deux la mesme violence. Je me tournay par après au troisième Manuscrit, (c'est celuy qu'ils appellent de Cave $\left.^{62}\right) \&$ ayant regardé attentivement la representation du moine Benedictin qui porte une croix en main, je n'ay pû me persuader que ce fust plustost l'Image de Jean Gersen, (comme l'a voulu l'Abbé Constantin qui l'a fait graver sur l'airain [en y ajoutant au dessous le nom de Gersen ${ }^{63}$ ]) que celle de sainct Benoist ou d'un autre Sainct, ou mesme d'un moine Benedictin pris indeterminement, dont le propre est de porter la croix de penitence, de la discipline, des jeunes \&c. veu principalement que dans les premieres pages de ce livre sont ces mots: Iste liber est Congregationis Cassinensis, \& peu après asseruatur in monasterio Cavae. Ce qui me fit aussi tost juger, ce livret estant sur la fin mutilé, sans nom d'Autheur, ny marque aucune de temps, que cette image avoit trompé l'Abbé Caëtan, \& qu'on ne pouvoit pas conclure par aucune raison probable, qu'elle fust plustost l'image de l'Autheur de ce livre, que de quelqu'autre Benedictin qui n'aura rien de commun avec cet autheur. [160] Je feüilletay enfin le quatrième livre, non pas Manuscrit, mais imprimé (c'est celuy qu'ils appellent de Genes $^{64}$ ). Et pour ce qui regarde l'escriture adjoustée à la fin, que ces Peres pretendoient avoir esté tracée avant qu'il y eut doute formé entre les Jesuites \& les Benedictins touchant l'Autheur du livre de l'Imitation, je trouvay que la chose alloit tout autrement, car elle est extrèmement nouvelle, $\&$ ce qui est de principal, c'est qu'elle est malade du mesme mal que le titre du second livre, pour ce qu'elle est fausse $\&$ suradjoustée à une autre plus ancienne, d'un artifice si grossier, que les ratures des mots apparoissent toutes entieres, sçavoir en certains vuides qui sont restez, \& les autres mots, qui y sont exprimez, donnent à connoistre le mal, ou au commencement ou à la fin. Et entre autres choses, du mot Thomas qui se lisoit en la premier écriture, on a formé si mal proprement qui soit esté, cét autre mot Ioannes, que Monsieur Vincenzo Galeotti Auditeur (comme on dit vulgairement) de Monseigneur l'Eminentissime Cardinal, venant à lire cette écriture, de bonne foy \& sans se tenir sur ses gardes pour quelque soupçon de fausseté, prononça Thomas, \& non pas Joannes, ce qui pouvoit m'arriver avant luy de mesme qu'à tout autre. Ayant fait mon rapport de toutes ces choses, à Monseigneur l'Eminentissime Cardinal, \& luy mesme ayant apperceu de ses

62 Codex Cavensis, Paris, BN, fonds Latin 13599.

${ }_{63} \mathrm{La}$ traduction proposée par Fronteau est à cet endroit lacunaire.

64 Venise, 1501, J. B. Sessa. 
propres yeux par une attentive inspection, que les choses estoient ainsi que je les avois remarquées dans les livres; il voulut neantmoins [161] que Monsieur Floravante en portast aussi son jugement, lequel s'estant rendu l'apres disnée en mon estude, \& ayant examiné les raisons \& les fondements de ces faussetez \& oppositions, ne pût rien trouver de contraire à mon jugement, mais se mit plustost à admirer la malice de ces personnes, qui bouleversent toutes choses pour contenter leurs affections \& soustenir des opinions qu'ils ont une fois conceuës. Qu'arriva-t'il enfin ? Les Peres Placide \& Jean vindrent aussi-tost, \& amenerent en leur compagnie le Notaire, avec la feüille cy attachée, pour recevoir la relation que Monsieur Floravante \& Moy devions faire en presence de Monseigneur le Cardinal de l'examen \& inspection de ces livres. Pour moy j'advertis le Pere Placide des faussetez, \& les ayant de nouveau examinez en presence aussi du Pere Jean, de Monsieur Floravante, \& mesme du Notaire, ils furent si manifestement convaincus de chacune fausseté en particulier, que lesdits Peres declarerent hautement, qu'ils n'avoient que faire qu'on dressast cét instrument par lequel leur cause seroit plustot perduë \& étouffée. Ce fut aussi le sentiment de Monsieur Vincenzo Galeotti Auditeur nommé, \& enfin celuy de Monseigneur le Cardinal de Bagny; que le Pere Placide alla voir à dessein seulement de sçavoir quelle estoit sa pensée touchant ces Manuscrits, \& laquelle il reconnût consister principalement à asseurer que ces Manuscrits ne contenoient rien de sain, rien de bon, ou de vray; aussitost le mesme Pere Placide ayant protesté auparavant qu'il n'avoit rien sceu de tant de faussetez \& resveries, recompensa le Notaire pour la moitié de son instrument, [162] comme s'il eust été entier, \& nous remercia tous du travail que nous avions diligemment employé, nous asseurant que doresnavant, ny les Peres Benedictins, ny aucune autre personne, ne pourroient l'engager à traiter de semblables affaires. Peu après Moy Gabriel Naudé, ayant retiré du Notaire ce qu'il avoit commencé à dresser de cét Acte, je crû qu'il estoit à propos d'y attacher au bas le narré de toute l'histoire, crainte que faute de la sçavoir, la verité de la question touchant l'Autheur des livres de l'Imitation de I. Chr. ne demeure opprimée, par des faussaires \& des trompeurs. Fait à Rome dans le Palais de l'Eminentissime Cardinal de Bagny le dernier jour de Janvier 1641.

Signé, Moy, Gabriel NAUDÉ le mesme que dessus.

Je Vincenzo Galeotti nommé cy-dessus confesse que tout ce qui est rapporté en cette presente écriture est veritable, \& que toutes ces choses se sont pasées comme elles sont racontées. En foy de quoy j'ay icy souscrit. À Rome, ce jour 16 septembre 1645.

VINCENZO GALEOTTI de ma main propre. 